\title{
AN OUTBREAK OF STREPTOCOCCAL INFECTION IN A MATERNITY UNIT
}

\author{
F. W. Nash, M.D.Lond., M.R.C.P., D.P.H.* \\ Trevor P. ManN, M.D.Lond., F.R.C.P., D.C.H.*
} Consultant Padiatricians to the Brighton and Lewes Group of Hospitals

I. W. Haydu, M. D. Pisa

Consultant Pathologist, Brighton and Lewes Group Hospital Laboratories

IN APRIL and May, 1962, an outbreak of infection due to Streptococcus pyogenes (Lancefield Group A.) occurred among newborn babies in a large communal nursery in one of the three wards of the maternity unit at the Brighton General Hospital. The responsible organism, which belonged to Griffith type 12 and was tetracycline-resistant, had only once previously been isolated in the Brighton area. We shall refer to it as the streptococcus.

Altogether, the organism was isolated from 5 adults and 19 babies. The adults were:-

2 pupil midwives who were symptomless carriers (positive throat swabs).

2 pupil midwives with tonsillitis.

1 mother with puerperal fever (positive high vaginal swab).

Considering next the 19 babies (11 males and 8 females), the streptococcus was isolated in the lying-in period in only 6 of them as follows:-

2 healthy babies, both umbilical carriers.

3 with a sticky umbilicus.

1 with a paronychia.

The streptococcus was isolated from 13 infants after their discharge from the unit as follows: -

1 with a fatal lung infection at the age of 16 days.

1 with a fatal septicæmia and peritonitis at the age of 4 weeks.

1 with a cellulitis of the face at the age of 26 days (positive mouth and throat swabs).

1 with purulent ophthalmia at the age of 3 weeks (this baby also had an opthalmia and a paronychia, both due to a penicillin-sensitive Staphylococcus pyogenes, which responded to treatment while in the ward). This was the only infant in the group developing : symptoms outside hospital who showed evidence of infection of any sort in the lying-in period.

* Address: Royal Alexandra Hospital for Sick Children, Dyke Road, Brighton.
2 with purulent otitis-both aged 7 weeks.

One of these infants had a combined infection with the streptococcus and a penicillinresistant Staphylococcus pyogenes.

Seven with a sticky umbilicus at ages ranging from 3-6 weeks.

Further details will now be given of 4 infected babies who showed features of special interest.

\section{Illustrative Case Reports}

Case No. 1. A male infant was born on April 29th. He was brought in dead to the Royal Alexandra Hospital, Brighton, on May 15th. He had been healtho and had appeared entirely normal until 24 hours. before his death when he became lethargic an refused his feeds. These symptoms worsened and the baby suddenly collapsed at home. Postmortem examination revealed a lung abscess from which the streptococcus was grown; there was nothing to suggest that the umbilicus had been the portal of entry.

Case No. 2. A female infant was born on April 17th. She was admitted to the Royal Alexandra Hospital in a moribund condition on May 15th, (aged 4 weeks) with a 5-day history of cough followed by diarrhœa and vomiting. Hitherto, she had been a healthy baby. On admission she was gravely ill (T. $96^{\circ} \mathrm{F}$. P.136) and presented a picture of severe dehydration and abdominal distension. There was some reddening and eversion of the umbilicus, and an extensive erythematous area could be seen over the right lower chest wall and the right side of the abdomen and flank. There were signs of free fluid in the peritoneal cavity. The baby was treated with intravenous fluids and given chloramphenicol and, later, penicillin by this route. A small amount of purulent flu:d was obtained by aspiration of the abdomen and from this the streptococcus was isolated. The infant died 12 hours after admission. Post-mortem examination demonstrated a generalized peritonitis with a collection of fluid pus in the lesser sac. A fusiform dilatation of the ligamentum teres was noted at its point of entry into the liver and there was marked thickening of its walls. On cutting across this structure pus escaped from both hepatic veins.

Case No. 3. A male infant was born on April 10th. He had been a normal infant and thrived well until four days before admission to the Royal Alex- 
andra Hospital on May 6th (aged 26 days). He presented with some swelling and redness of the skin of the left side of the neck which spread to involve the left maxillary region. He had been treated at home with tetracycline, $62.5 \mathrm{mg}$. 6-hourly, for three days without any improvement. On admission the baby's general condition was good; he was febrile (T. $102.8^{\circ} \mathrm{F}$.) and had marked inflammatory cedema of the left eye-lids and face. Blood culture was sterile. A throat swab on admission grew the streptococcus and the same organism was isolated from the mouth. He was thought at first to have a staphylococcal osteitis of the left maxilla but it soon became apparent that the baby was suffering from streptococcal cellulitis. He was treated with erythromycin, $25 \mathrm{mg}$. 6-hourly, for nine days with good response.

Case No. 4. A female infant was born on April 13 th. She was quite healthy until the age of seven weeks (6 weeks after discharge from the maternity unit) when she developed a right purulent otitis. The streptococcus was grown from an ear swab and the baby treated with penicillin-at first by mouth for 3 days and then intramuscularly, for 5 days. On June 1 st (8 days after commencing treatment) an ear swab was still positive for the streptococcus and, on this occasion, a penicillin-resistant staphylococcus pyogenes was also grown. The discharge had lessened but, on June 7 th, it became more evident again. At this point the infant was admitted to the Royal Alexandra Hospital and was found to have a purulent otitis media with perforation of the right tympanic membrane. Another swab of the pus grew the streptococcus and, once more, the penicillinresistant Staphylococcus pyogenes. Treatment with intramuscular penicillin and oral chloramphenicol for 7 days lead to a cessation of discharge; nevertheless on June 13th an ear swab still produced a scanty growth of the two organisms.

\section{Management of the Epidemic}

In spite of extensive swabbing of patients and staff we were unable to determine precisely when and how the streptococcus gained entrance to the nursery. However, in retrospect it seems likely that "bacteriological" crossinfection with the epidemic organism had been occuring for some time before we appreciated what was happening. A surprising feature of the outbreak was the fact that it involved only the one nursery, although there were ample opportunities at the time for wide dissemination of the infection to take place. For one thing, there was a constant interchange of nursing staff between the three wards and their nurseries.

The organism was first isolated on April 18 th, 1962, from the slightly moist umbilicus of an otherwise healthy baby and soon afterwards it became apparent that we were dealing with a severe nursery epidemic. The outbreak, once recognized, was quickly brought under control with the help of the following measures:
1. Nose and throat swabs were taken from everyone in the Unit and umbilical swabs from all babies, on the fourth and eighth days of life. Those individuals shown to be harbouring the streptococcus were isolated.

2. The affected ward was closed to new cases, emptied as quickly as possible by the early discharge of mothers and babies under careful medical surveillance at home and then fumigated.

3. The administration in appropriate dosage of sodium benzylpenicillin as follows: - the crystalline preparation 12-hourly by the intramuscular route for five days to those adults and babies from whom the streptococcus was isolated; phenoxymethyl penicillin by mouth for one week to all infants in the Maternity Department whose nose, throat and umbilical swabs were negative.

4. Nursery techniques then in use were carefully scrutinized and several changes made in the routine. These included restricting the handling of infants to a minimum, eliminating some objects in communal use (notably the changing table) and enforcing a strict handwashing ritual. We also decided to modify the routine care of the umbilical cord along the lines shown by the Bristol workers to be effective in controlling staphylococcal sepsis amongst newborn infants in a maternity hos:pital (Simpson, Tozer and Gillespie, 1960).

5. With the co-operation of local Medical Officers of Health and general practitioners all babies known to be at risk were followed up in their homes and bacteriological cultures were taken from any infant recently discharged from hospital who developed signs of an infection.

\section{Discussion}

Infection of the newly born infant is one of the major disadvantages of hospital midwifery and is part of the general problem of hospital cross-infection. The commonest pathogenic organism is Staphylococcus pyogenes, which is now endemic in most, if not all, hospitals. In contrast, Streptococcus pyogenes, although at one time an important cause of infection in obstetric units (Colebrook and Kenny, 1936), is now not often encountered. However, our experience with this streptococcal outbreak is not unique and there are a number of reports in the literature demonstrating how rapidly and unexpectedly a maternity unit may be invaded by this pathogen, leading to deaths in neonates from overwhelming infection (Boissard 
and Eton, 1956; Gibson and Calman, 1953; Gray, 1956; Kwantes and James, 1956; Langewisch, 1956; Nicol, 1961).

Streptococcus pyogenes infections due to a tetracycline-resistant organism have, until comparatively recently, been uncommon. This type of streptococcus had only been identified once in the Brighton area before this outbreak; since then it has been encountered with increasing frequency. Thus, figures from the Brighton Group Laboratories show that over the past year (March 1963-April 1964) Streptococcus pyogenes has been isolated from inpatients on 276 occasions and that no less than $47 \%$ of these cultures have been resistant to tetracycline, an experience paralleled by others (Kuharic, Roberts and Kirby, 1960; Lowbury and Hurst, 1956; Mogabgab and Pelon, 1958; Parker, Maxted and Frazer, 1962).

This severe outbreak of cross-infection illustrates several important clinical and epidemiological features of Streptococcus pyogenes infection in the newborn infant, confirming the experience of others. These may be summarized as follows: -

1. Streptococcus pyogenes, like Staphylococcus pyogenes, can colonize the umbilicus shortly after birth without producing any visible local reaction or giving rise to any illness.

2. Once umbilical colonization has occurred the organism may lie dormant in the deeper tissues of a healthy baby and lead to a fulminating septicæmia several weeks later, when the baby's association with the hospital has almost been forgotton. In this connection it is worth noting that in normal babies unorganized blood clot may be visible in the umbilical vessels for as long as 50 days after delivery and fibrous occlusion may take longer (Chamberlain, 1936).

3. A combined infection with a penicillinresistant Staphylococcus pyogenes may render a Streptococcus pyogenes infection resistant to the administration of penicillin given in conventional dosage presumably due to inactivation by penicillinase. This is well illustrated in the case of Baby D. with otitis media, described above. The same phenomenon has been observed by Gray (1956).

The authors are indebted to Dr. P. VassalloAgius for his help with organizing the data which form the basis of th:s report. Our thanks are also due to the local Medical Officers of Health and to Dr. J. E. Jameson, Director of the Brighton Public Health Laboratory, for their assistance with bacteriological studies performed on infants in their homes.

\section{REFERENCES}

Boissard, J. M., and Eton, B. (1956): Neonatal Umbilicus as a Source of Streptococcal Infections in a Maternity Unit, Brit. med. J., ii, 574.

Colebrook, L., and KenNy, M. (1936): Treatment with Prontosil of Puerperal Infections, Lancet, ii, 1319. Chamberlain, J. W. (1936): Omphalitis in the Newborn, J. Paediat, 9, 215.

Gibson, J., and Calman, R. M. (1953): An Outbreak of Streptococcal Infection in a Maternity Hospital, Lancet, i, 320.

Gray, J. D. Allen (1956): Outbreak of Streptococcal Infection in a Maternity Hospital, Lancet, ii, 132.

Kuharic, H. A., Roberts, C. E., and KIRBY, W. M. M. (1960): Tetracycline Resistance of Group A Beta Hemolytic Streptococci, J. Amer. med. Ass., 174, 1779.

KWANTES, W., and James, J. R. E. (1956): Hæmolytiळ बै Streptococci on the Neonatal Umbilicus, Brit. medo J., if, 576.

LANGEWISCH, W. H. (1956): An Epidemic of Group A, Type 1, Streptococcal Infections in Newborn Infants, Padiatrics, 18, 438.

Lowbury, E. J. L., and Hurst, L. (1956): Atypical Anærobic Forms of Streptococcus Pyogenes associated with Tetracycline Resistance, J. clin. Path., 9, 59.

Mogabgab, W. J., and Pelon, W. (1958): An Outbreak of Pharyngitis due to Tetracycline-Resistant Group A, Type 12 Streptococci, Amer. J. Dis. Child, 96, 696.

Nicol, C. G. M. (1961): Monthly Bulletin Ministry of Health and Public Health Lab. Service, 20, 68.

PARKer, M. T., MAXTED, W. R., and Frazer, C. A. M. (1962): Letter. Tetracycline-Resistant Hæmolytic Streptococci, Brit. med. J., ii, 1550.

Simpson, K., Tozer, R. C., and Gillespie, W. A (1960): Prevention of Staphylococcal Sepsis in a Maternity Hospital by means of Hexachlorophane, ibid, i, 315. 